\title{
Fratura Supracondiliana do Fêmur \\ Durante Salto Após Reconstrução \\ Artroscópica do Ligamento \\ Cruzado Anterior
}

\author{
Supracondylar Femur Fracture During Jump After Anterior \\ Cruciate Ligament Arthroscopic Reconstruction
}

Thiago Yukio Fukuda',

Paula Meletti Yazbek',

Flavio Fernandes Bryk'

1.Setor de Fisioterapia / Irmandade da Santa Casa de Misericórdia de São Paulo / São Paulo, SP.

\section{Endereço para correspondência:}

Thiago Yukio Fukuda

Rua Doutor Cesário Mota Jr., 112 -01221-900 - São Paulo, SP- Brasil.

Tel.: (55-11) 2176-7000 ramal 1591.

E-mail: tfukuda@scamilo.br

Submetido em 30/10/2008 Versão final recebida em 04/11/2008 Aceito em 08/01/2009

\begin{abstract}
RESUMO
Contextualização: A fratura distal do fêmur é uma das possíveis complicações no período pós-operatório de reconstrução de LCA, porém, de incidência rara. Relato de caso: Descreve-se o caso de um atleta de 34 anos, gênero masculino, no quinto mês de pós-operatório de reconstrução de LCA. O caso evoluía normalmente de acordo com o protocolo estabelecido; o paciente apresentava bom controle e estabilidade sensoriomotora, quando sofreu fratura supracondiliana do fêmur ao realizar um salto durante atendimento fisioterápico. A reconstrução ligamentar foi realizada com enxerto dos músculos flexores do joelho e a fratura, ocasionada posteriormente, reduzida e fixada com placa e parafuso. Dez meses após a redução aberta e fixação interna da fratura, apresenta função regular na escala Lysholm, amplitude de movimento normal e força muscular grau V em flexores e extensores da coxa. Discussão: Poucos relatos de caso semelhantes ao presente foram encontrados na literatura; a maioria apresentou fratura do fêmur após a reconstrução do LCA com tendão patelar. Este caso mostra-se relevante pela associação da fratura supracondiliana do fêmur com reconstrução ligamentar com tendões dos flexores, visto que apenas um trabalho seguiu tal direção. Dentre as prováveis causas dessa fratura, destacam-se uma fragilidade óssea por desuso e túnel ósseo femoral de diâmetro maior que o padrão, apesar de não haver consenso em relação a essas alterações. Uma hipótese sugerida pelos autores deste relato é de que o túnel ósseo de fixação do enxerto pode ter sido um intensificador de estresse sobre o local da fratura.
\end{abstract}

Palavras-chave: complicação, cirurgia, joelho.

\section{ABSTRACT}

Background: Distal femoral fracture is one of the possible complications on the post operative period of the $A C L$ reconstruction; however, with rare incidence. Case report: This study reports a male 34 year-old athlete, five months after ACL reconstruction surgery. The case developed normally in accordance with the established protocol. The patient presented good control and sensory-motor stability when he suffered a supracondylar femur fracture. This fracture occurred during a jump in the physical therapy treatment. The ligament reconstruction was done with the tendon graft of the knee flexors muscles, and the fracture caused later, was reduced and stabilized with plate and screw. Ten months after open reduction and internal fixation of the fracture, the patient showed regular function in the Lysholm scale, normal range of movement and muscular force grade $V$ for knee flexors and extensors. Discussion: Few case reports similar to the present one were found in the literature. The majority presented femur fracture after ACL reconstruction with patellar tendon. The present case shows significance by the association between supracondylar femur fracture with ligament reconstruction with flexors tendon, since only one case followed this direction. Two of the possible causes of this fracture are bone fragility for disuse and femoral tunnel bone bigger than the normal, despite not being a consensus. A hypothesis suggested by the authors of this paper is that the bone tunnel of graft fixation could have been a stress booster to the site of the fracture.

Keywords: complication, surgery, knee. 


\section{INTRODUÇÃO}

A reconstrução do ligamento cruzado anterior (LCA) é comumente realizada com a utilização de aloenxertos, resultando em expectativas cada vez maiores em relação à reabilitação e ao retorno precoce às atividades esportivas $^{(1)}$. Durante o programa de reabilitação, utiliza-se o hop test como uma forma diagnóstica da função motora pós-operatória associado a exercícios pliométricos em etapas avançadas, em torno de quatro meses após cirurgia ${ }^{(2)}$. Tal teste pode ser também empregado como critério de evolução com objetivo de mensurar a estabilidade dinâmica articular, força e coordenação(3).

Dentre as possíveis complicações no período pós-operatório de reconstrução do LCA, a fratura supracondiliana raramente ocorre. Segundo escassos relatos de caso(4-6) encontrados na literatura, esse tipo de fratura ocorreu após reconstrução com enxerto proveniente do terço médio do tendão patelar, em sua maioria.

O caso apresentado é uma fratura supracondiliana femoral durante salto em single hop test no período de cinco meses de pós-operatório de reconstrução de LCA. Trata-se de uma fratura transversa de diáfise femoral distal, no nível do túnel intraósseo femoral utilizado para fixação do enxerto. O paciente assinou Termo de Consentimento Livre e Esclarecido em 30 de junho de 2008 e esse relato foi aprovado pelo Comitê de Ética em Pesquisas em Seres Humanos da Irmandade da Santa Casa de Misericórdia de São Paulo, em 13 de agosto de 2008, projeto n004/08.

\section{RELATO DO CASO}

Paciente do gênero masculino, atleta, 34 anos, sofreu entorse em joelho direito durante uma partida de basquete em setembro de 2006 e foi admitido no serviço da ISCMSP uma semana após o ocorrido. Submeteu-se a todos os exames clínicos e de imagem pertinentes, sendo diagnosticada ruptura completa do LCA direito associada à lesão meniscal medial do tipo alça de balde.

Em março de 2007 foi submetido a cirurgia por via artroscópica para reparo desse ligamento com uso de enxerto dos tendões semitendíneo e grácil e fixação através de parafusos de interferência distal e proximal, além de meniscectomia parcial. Deu início à fisioterapia ambulatorial uma semana após alta hospitalar, com seguimento de protocolo utilizado pelo Serviço de Reabilitação da Santa Casa de São Paulo baseado em reequilíbrio biomecânico e sensoriomotor, respeitando as fases de cicatrização do neoligamento.

No terceiro mês de tratamento, paciente apresentou uma distensão muscular em semimembranaceo, evoluindo com quadro álgico intenso e culminando em alteração e regressão de conduta fisioterápica. Com cinco meses de pós-operatório, o paciente realizou, pela primeira vez, saltos com apoio unipodal (hop test). Em agosto de 2007, aproximadamente cinco meses e 15 dias de pós-operatório de reconstrução de LCA, o paciente referiu dor súbita e incapacitante acima do joelho direito após um dos saltos. Foi encaminhado para exame radiográfico, sendo diagnosticada fratura supracondiliana em fêmur direito tipo 33A1, de acordo com a Classificação AO para fraturas do fêmur distal (figura 1).

No dia seguinte ao do episódio de fratura, foi realizado reparo cirúrgico com redução aberta e fixação interna com placa e parafuso do tipo DCS (dinamic condylar screw) (figura 2).

No período de uma semana após fixação da fratura, retomou tratamento fisioterápico ainda em enfermaria hospitalar, com conduta baseada em eletroestimulação neuromuscular e exercícios para ganho de força muscular e amplitude de movimento articular.

Em janeiro de 2008, cinco meses após a fratura, foi submetido a novos exames radiográficos, nos quais foram observados sinais de consolidação (figura 3). O paciente foi submetido a uma série de ques-

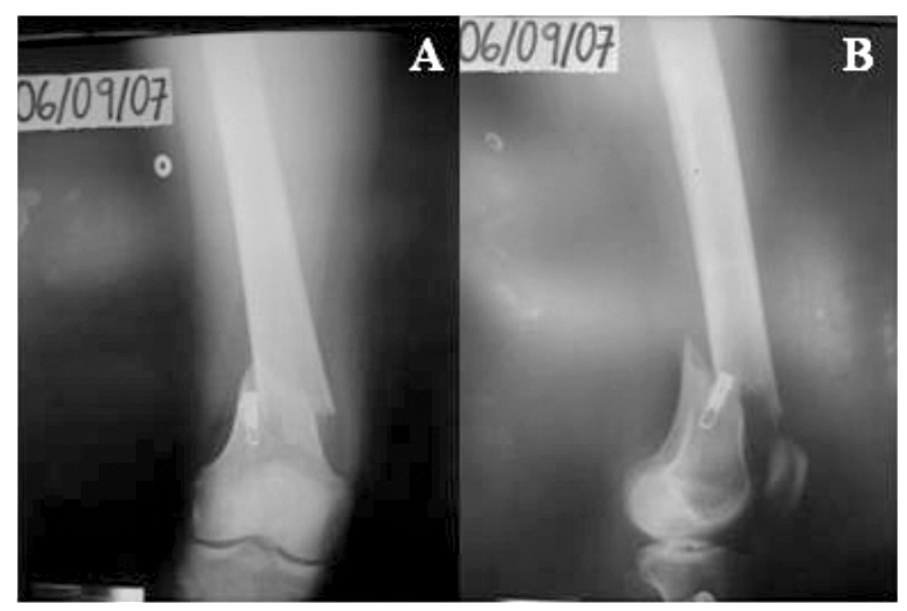

Figura 1. Radiografia ântero-posterior (A) e perfil (B) exibindo fratura supracondiliana

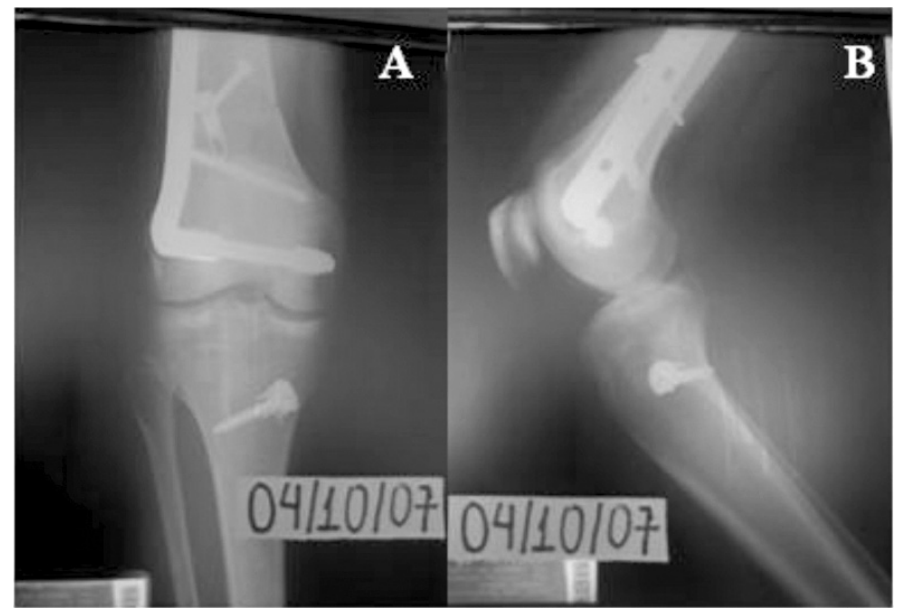

Figura 2. Radiografia ântero-posterior (A) e perfil (B) exibindo fixação interna de fratura supracondiliana femoral com placa e parafuso do tipo DCS

tionários para evolução de conduta de seu tratamento e planejamento de retorno às atividades de vida diária. Em follow-up de 10 meses após redução aberta e fixação interna do fêmur, ainda em acompanhamento fisioterápico, encontrava-se com ADM ativa e passiva em flexão de joelho de $122^{\circ}$ para perna direita e $124^{\circ}$ para a esquerda, força muscular grau $\mathrm{V}$ em extensores e flexores de perna e abdutores, extensores e flexores de coxa, escala de Lysholm com pontuação 65 (regular) e queixa álgica próximo às protuberâncias dos parafusos.

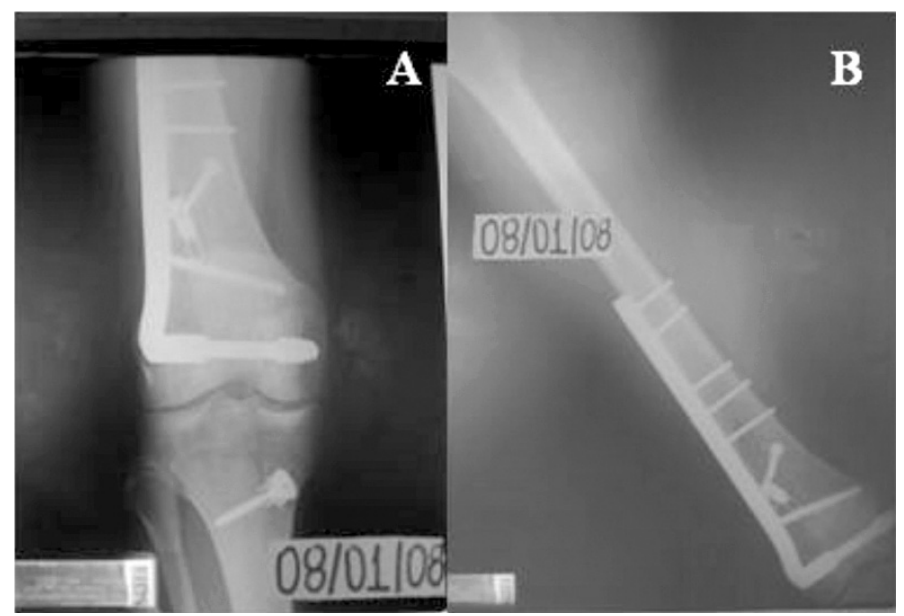

Figura 3. Radiografia ântero-posterior (A) e perfil (B) exibindo sinais de consolidação em foco da fratura 


\section{DISCUSSÃO}

A incidência de fratura distal do fêmur após reconstrução de LCA é rara. Foram encontrados poucos relatos semelhantes ao do presente e, dentre eles, apenas quatro $\operatorname{casos}^{(1,7,8)}$ utilizaram enxerto de tendão dos flexores para reconstrução do LCA. Quanto ao local da fratura, apenas Noah et al.(4), Ternes et al.(5), Radler et al.(6) e Sheps et al.(8) relataram fraturas supracondilianas como complicação de reconstrução de LCA. Entretanto, dentre todos os casos descritos até o momento, apenas Sheps et al. ${ }^{(8)}$ descrevem caso com associação de fratura supracondiliana do fêmur e reconstrução de LCA com enxerto de tendão de flexores de perna. Nenhum dos casos encontrados na literatura ocorreu durante a realização de salto.

Segundo Arriaza et al. (7), a combinação de fixação transversa femoral e programa de reabilitação acelerado pode ser uma possível causa da fratura supracondiliana após reconstrução do LCA. Sugere-se que o mecanismo de fratura esteja associado à queda com o joelho parcialmente flexionado, causando impacto do côndilo tibial superiormente sobre parte posterior do côndilo femoral ${ }^{(1,9)}$.

Outras causas relacionadas com fraturas femorais pós-reconstrução de LCA envolvem, em sua maioria, fatores intensificadores de estresse, incluindo os orifícios dos parafusos de interferência. Burstein et al.(10) relataram que o orifício de um parafuso recém-aplicado ao osso faz com que haja fraqueza de sustentação de carga e torção. Esse orifício também influi na localização de fraturas em espiral secundárias, uma vez que, em $90 \%$ dos casos, a linha da fratura passa através do orifício. A fraqueza tende a ser da mesma magnitude para qualquer orifício que envolva menos do que 30\% do diâmetro ósseo. Sheps et al( ${ }^{(8)}$ acrescentam que cuidados devem ser tomados no intra-operatório para não perfurar o córtex ântero-lateral distal do fêmur mais do que o necessário. Apesar de todos esses fatores, a magnitude de concentração de estresse sobre o túnel ósseo é biomecanicamente desconhecida ${ }^{(11)}$.

Adicionalmente, ruptura do LCA tratada cirurgicamente implica considerável perda óssea mineral no joelho afetado, principalmente na região distal do fêmur. Podem ser citados pelo menos três fatores que influenciam tal ocorrência: o trauma por si só, aumentando níveis hormonais responsáveis pelo direcionamento mineral; o próprio procedimento cirúrgico, que interfere na estrutura óssea; e a possível imobilização pós-traumática, levando ao desuso do membro(12).

O protocolo de reabilitação aplicado pela equipe responsável no presente estudo respeita o tempo fisiológico necessário para cicatrização do enxerto. Dessa forma, a realização de saltos é iniciada em torno do quarto mês pós-operatório ${ }^{(13)}$.

A lesão relatada nesse caso envolveu um jovem com boa qualidade óssea, no quinto mês de pós-operatório de uma bem-sucedida reconstrução do LCA, ilustrando um problema de aparecimento tardio de instabilidade óssea distal do fêmur inesperadamente. Esse caso é particularmente significante, uma vez que o túnel ósseo para fixação do enxerto pode ter sido um intensificador da fragilidade local adicional.

Todos os autores declararam não haver qualquer potencial conflito de interesses referente a este artigo.

\section{REFERÊNCIAS BIBLIOGRÁFICAS}

1. Manktelow ARJ, Haddad FS, Goddard NJ. Late lateral femoral condyle fracture after anterior cruciate ligament reconstruction. A case report. Am J Sports Med. 1998;26(4):587-90.

2. Noyes FR, Barber SD, Mangine RE. Abnormal lower limb symmetry determined by function hop tests after anterior cruciate ligament rupture. Am J Sports Med. 1991;19(5):513-18.

3. Myer GD, Paterno MV, Ford KR, Quatman CE, Hewett TE. Rehabilitation after anterior cruciate ligament reconstruction: Criteria-based progression through the return-to-sport phase. J Orthop Sports Phys Ther. 2006;36(6):385-402.

4. Noah J, Sherman $\mathrm{OH}$, Roberts $\mathrm{C}$. Fracture of the supracondylar femur after anterior cruciate ligament reconstruction using patellar tendon and iliotibial band tenodesis. Am J Sports Med. 1992;20(5):615-8.

5. Ternes JP, Blasier RB, Alexander AH. Fracture of the femur after anterior cruciate ligament reconstruction with a GORE-TEX prosthetic graft. Am J Sports Med. 1993;21(1):147-9.

6. Radler C, Wozasek GE, Seitz H, Vécsei V. Distal femoral fracture through the screw hole of a ligament augmentation device fixation. Arthroscopy. 2000;16(7):737-9.

7. Arriaza R, Señaris J, Couceiro G, Aizpurua J. Stress fracture of the femur after ACL reconstruction with transfemoral fixation. Knee Surg Sports Traumatol Arthrosc. 2006;14:1148-50.
8. Sheps DM, Reed JG, Hildebrand KA, Hiemstra LA. Supracondylar femur fracture after endoscopic anterior cruciate reconstruction using an EndoButton. Clin J Sport Med. 2006;16(5):428-9.

9. Wilson TC, Rosenblum WJ, Johnson DL. Fracture of the femoral tunnel after an anterior cruciate ligament reconstruction. Arthroscopy. 2004;20(5):e45-7.

10. Burstein AH, Currey J, Frankel VH, Heiple KG, Lunseth P, Vessely JC. Bone strength. The effect of screw holes. J Bone Joint Surg Am. 1972;54(6):1143-56

11. Berg EE. Lateral femoral condyle fracture after endoscopic anterior cruciate ligament reconstruction. Arthroscopy. 1994;10(6):693-5

12. Leppälä J, Kannus P, Natri A, Pasanen M, Sievänen H, Vuori I, et al. Effect of anterior cruciate ligament injury of the knee on bone mineral density of the spine and affected lower extremity: A prospective one-year follow-up study. Calcif Tissue Int. 1999;64:357-63.

13. Beynnon BD, Uh BS, Johnson RJ, Abate JA, Nichols CE, Fleming BC, et al. Rehabilitation after anterior cruciate ligament reconstruction - A prospective, randomized, double-blind comparison of programs administered over 2 different time intervals. Am J Sports Med. 2005;33(3):347-58. 